\title{
Cost-effectiveness and Diet Quality in a 3-year Follow-up of a Randomised Controlled Trial (The BALANCE trial)
}

\author{
Camila Ragne Torreglosa ( $\square$ camilatorreglosa@gmail.com ) \\ Universidade de São Paulo https://orcid.org/0000-0002-9385-0148 \\ Flávia Mori Sarti \\ Universidade de Sao Paulo \\ Ângela Cristine Bersch Ferreira \\ Hospital do Coracao \\ Bemardete Weber \\ Hospital do Coracao \\ Renato Hideo Nakagawa Santos \\ Hospital do Coracao \\ Murilo Mazzotti Silvestrini \\ Universidade de Sao Paulo \\ Alexandre Dias Porto Chiavegatto Filho \\ Universidade de Sao Paulo
}

\section{Research article}

Keywords: Cost, Cost-effectiveness, diet quality, dietary pattern, cardiovascular disease.

Posted Date: July 30th, 2020

DOl: https://doi.org/10.21203/rs.3.rs-49300/v1

License: (c) (1) This work is licensed under a Creative Commons Attribution 4.0 International License. Read Full License 


\section{Abstract}

Background: A healthy diet is essential to reduce cardiovascular disease (CVD) risk and mortality. However, recent studies lack conclusive evidence on the affordability of cardioprotective diets worldwide. The Brazilian Cardioprotective Nutritional Program Trial (BALANCE Program) is a randomized multicenter clinical trial that proposes regionally adapted cardioprotective diet that achieves nutritional recommendations and incorporates accessible and affordable foods. The study aim is to analyze cost-effectiveness of the BALANCE Program in comparison to generic dietary advice for individuals, based on diet costs and nutritional quality among patients with high adherence to the study protocol in both control and intervention groups.

Methods: We conducted a cost-effectiveness analysis of subsample from the BALANCE Program (1,161 individuals with previous event of CVD and high adherence to study protocol enrolled in 35 research sites throughout Brazil) after a 3-year follow-up. Direct costs and nutritional quality of diets reported by participants were estimated at the individual level. Diet costs were based on market prices collected from five major supermarket chains. Effectiveness was measured in terms of diet quality, according to adherence to the Brazilian Health Eating Index Revised (BHEl-R). Mean differences were compared between groups using generalized estimating equation. Monte Carlo simulations were performed to comprise probabilistic sensitivity analysis regarding trends in the comparison between groups.

Results: At baseline, mean direct diet costs were equal (U\$3.9/day), and there were small differences in BHEI-R between groups (53.5 points in BALANCE Program, and 51.8 points in control group). After the 3-year follow-up, the intervention was associated with a mean cost saving of U\$0.31/day ( $95 \% \mathrm{Cl}$ : $-0.59 ;-0.04)$ and mean BHEI-R increase of 4.38 (95\% Cl: $2.81 ; 5.95)$.

Conclusion: The intervention was dominant strategy in terms of cost-effectiveness due to higher effectiveness at lower costs in comparison with the control group.

Conclusions: There was a statistically significant effect of the intervention regarding increase in diet quality and reduction in cost after 3 years compared to the control group. The BALANCE intervention may be an option to improve diet quality with lower costs among Brazilians with CVD.

Trial registration: NCT, NCT01620398. Registered June 15, 2012. https://clinicaltrials.gov/ct2/show/NCT01620398

\section{Background}

Cardiovascular disease (CVD) is a leading cause of death and disability worldwide, and also in Brazil(1,2). Secondary prevention programs with focus on management of cardiovascular risk factors have been associated with substantially lower risk of recurrent cardiovascular events ${ }^{(3,4)}$. Additionally, the adoption of healthy behaviors (e.g., diet, exercise, smoking cessation, etc.) is recommended by international guidelines ${ }^{(5,6)}$. There are many studies indicating that specific combinations of foods and dietary patterns contribute to prevent new events and to control CVD risk factors(7,8).

According to current epidemiologic evidence, food-based measures to reduce CVD risk and mortality include an optimal intake of whole grains, fruits, vegetables, legumes, nuts, seeds, fish and reduced consumption of red and processed meat ${ }^{(9,10)}$. Although this is considered the best nutritional recommendation, health professionals must ponder that food availability is highly diverse worldwide, so dietary advice might fail without personal tailoring. One potential explanation for that failure could be the high food expenditures resulting from the intake of non-locally-produced foods ${ }^{(11)}$. Furthermore, food prices directly influence food choice and are among the main barriers to dietary changes ${ }^{(12)}$.

Overall, whether the cost of a cardioprotective diet is higher or lower compared with a conventional diet remains unclear. Observational studies have shown that cardioprotective dietary patterns (DASH or Mediterranean diet) are more expensive than usual local diets ${ }^{(13-15)}$. In addition, the adherence to a Mediterranean dietary pattern in non-Mediterranean population could cost $24 \%$ more ${ }^{(14)}$. Recently, there have been efforts to develop a regional adaptations of the Mediterranean diet in studies with non-Mediterranean populations ${ }^{(16-18)}$. Once the diet is adapted, it is possible to increase its affordability, reaching the same price as the conventional diet ${ }^{(16,17)}$.

Brazilian Cardioprotective Nutritional Program Trial (BALANCE Program) is a multicentre randomised controlled trial designed to investigate the effects of an educational intervention aimed at improving dietary habits of patients with previous CVD. The intervention has the main purpose of developing a Brazilian cardioprotective diet that achieves standard nutritional recommendations for treatment of CVD and, concurrently, incorporates local food products to respect regional habits, multi-cultural factors and affordability. The main results of the BALANCE Program were previously published ${ }^{(19-21)}$ showing diet quality improvement after a 48-months follow-up, but no differences in mortality or cardiovascular events.

In this study, the objective was to analyze cost and diet quality and to perform a cost-effectiveness analysis of the BALANCE Program in comparison with generic dietary advice (control group) in individuals with high adherence to study protocol.

\section{Methods}

\section{Study design and participants}

The study design is a retrospective analysis of 24-hour recalls from participants of the BALANCE program study (https://www.clinicaltrials.gov/; NCT01620398). A description of the original study has been published elsewhere ${ }^{(20)}$. 
Briefly, between March 2013 and January 2015, 2534 individuals who experienced one or more indicators of established CVD in the preceding 10 years were enrolled in the trial and followed until December 2017 in one of the 35 research sites in Brazil. They were then randomised to either the control group or the intervention group (BALANCE Program). The study was approved by the Research Ethics Committee of the Hospital do Coração de São Paulo and all participants signed an informed consent form.

The participants in the control group received generic nutritional advice on low-fat, low-energy, low-sodium, and low-cholesterol diet. The participants in the intervention group had frequent contact with registered dietitians and received diet prescriptions based on the Brazilian dietary guidelines for treatment of $C V D^{(22)}$, including specific educational intervention for improvement in dietary patterns, especially regarding the consumption of locallyavailable foods with cardioprotective role (i.e., $50 \%$ to $60 \%$ of energy from carbohydrate, $10 \%$ to $15 \%$ from protein, $25 \%$ to $35 \%$ from total fat, $7 \%$ from saturated fatty acids, $10 \%$ polyunsaturated fatty acids, $20 \%$ monounsaturated fatty acids, $1 \%$ trans fats, $200 \mathrm{mg} / \mathrm{day}$ cholesterol, 20 to $30 \mathrm{~g} / \mathrm{d}$ fiber, and $2400 \mathrm{mg} / \mathrm{d}$ sodium). Trained registered dietitians provided individualized dietary advice (face-to-face or telephone sessions) for each participant.

For the present study, per-protocol analysis was carried out with adherence to protocol being defined as $80 \%$ presence in the individual sessions and telephone calls during the 36 months of follow-up. The adherence cut-off was defined considering prior studies that demonstrated higher treatment compliance due to closer contact with health professionals ${ }^{(23,24)}$. The final sample consisted of 1,161 individuals (576 in the intervention group and 585 in the control group, $40.2 \%$ of the original sample).

Dietary intake data from 24-hour recalls were used to estimate diet costs and quality. Trained researchers collected five 24-hour recall during the 36 followup months: two at the beginning of the study (before the intervention with a 15-day interval between them), and three in the following years, once a year.

\section{Cost-effectiveness analysis}

The economic assessment for comparison of the two strategies (control and BALANCE Program) was performed based on cost-effectiveness ratios (CER) and incremental cost-effectiveness ratios (ICER). The analyses were conducted using the perspective of the patients. This perspective was adopted because patients are directly affected by the intervention, and decide to follow or not the prescribed diet. The time horizon was established in 36 months, based on health outcomes presented in the trial paper previously published. ${ }^{(21)}$ Costs were valued in the same time period so the discount rate was not applied

Effectiveness was assessed using diet quality from the Brazilian Health Eating Index Revised (BHEI-R) ${ }^{(25)}$, and direct costs were calculated from food items reported during the 24-hour recall interviews. The direct costs to perform the intervention (e.g., researchers wages, transport of subjects to study centers, biochemical analyses, etc.) were not included in the analysis, considering that the program was designed to comprise a strategy for secondary prevention at primary health care level; therefore, should be implemented in local settings near individuals' residence.

\section{Estimation of diet costs}

The cost of the diet was assessed at individual level, based on a dataset of food items reported by the participants. The dataset was constructed by compiling a full list of food items from each of the 24-hour recalls, resulting in 1,103 standardized food items and recipes.

Prices of food items were collected between October and December 2018 in three local supermarkets located in the Northeast, Midwest and South of Brazil, and two nationwide supermarket chains with online stores. Prices were registered for usual retail purchase of food items, promotions referring to sales or bulk acquisition were discarded during consistency analysis. In the case of more than one product available of the same food, data were collected from up to three items, and the average price of the items was used as the final price.

The price of each raw food was converted into price per gram of food ready to eat by applying correction and cooking factors. The recipes were obtained from the BALANCE Program recipes book or a Brazilian standard book for recipes ${ }^{(21)}$, using the amount of main ingredients in the recipes, and including standardized amounts of seasonings (e.g., $5 \%$ of sugar, $1 \%$ of salt and $2 \%$ of soy oil for cooked or $10 \%$ for fried foods), in order to allow its inclusion in costs. Prices of food items were deflated to the period of 24-hour recall interviews using official data on specific inflation rates for each item at local level, in order to properly represent relative prices of purchase at the time of the interview, as published by the Brazilian Institute for Geography and Statistics $(\text { IBGE) })^{(26)}$

Finally, the cost of the diet was estimated by multiplying the mean price per gram of the food item by the amount reported in the 24 -hour recall for each participant. The cost of the diet was updated and converted into U.S. dollars using the official exchange rate published by the Brazilian Central Bank at the reference date of December, 2018 (1 US dollar = 3.88 Brazilian Reais).

\section{Assessment of diet quality}

The BHEl-R ${ }^{(25)}$ is a validated adaptation of the Healthy Eating Index 2005 for the Brazilian population ${ }^{(27)}$, based on nutrition recommendations from the Brazilian Ministry of Health Food Guide, the World Health Organization, the Institute of Medicine, and the guidelines of the Brazilian Society of Cardiology (27). 
The BHEI-R score for each 24-hour recall was obtained by the sum of scores referring to twelve components: nine based on the consumption of food groups (total cereals; whole grains; total fruits; whole fruits; vegetables; dark green and orange vegetables and legumes; milk and dairy products; meats, eggs and legumes and oils), two based on the intake of nutrients (saturated fat and sodium), and one resulting from the energy intake from solid fat, alcohol, and added sugar (SoFAAS). Each component can contribute from 0 to 20 points to the total score, depending on component type (food group or nutrient intake). Minimum to maximum values were determined according to the nutritional recommendations of each component based on national and international guidelines; e.g., in the case of the group "total fruit", dietary intake equal to or greater than the recommended per 1,000 kcal was given the maximum score of the item (five points).

Nutritional values of 24-hour recalls were calculated using the Nutriquanti software, and BHEI-R score, ranging from 0 to 100 points, was considered the outcome variable in the economic assessment and in the statistical analysis. Higher BHEI-R values indicate better dietary quality, whereas low scores indicate less adherence to recommendations.

\section{Statistical analysis}

Between-group differences in baseline characteristics were analyzed with the Wilcoxon rank-sum test for continuous variables and chi-square for categorical variables. Regarding costs and diet quality, mean differences at baseline and post-intervention (1, 2 and 3 years later) were compared between groups using generalized estimating equation (GEE) models with unstructured and exchangeable correlation matrix, adopting time, treatment, and interaction between time and treatment as predictors.

Differences in scores of BHEI-R components, total energy, total amount of food, amount of food group, costs, energy density, and macronutrients between the intervention and control groups at end point were analysed using ANCOVA, with baseline variables as covariates. Mean difference was presented as difference between the BALANCE and the control group.

Analyses considered a 2-tailed statistical significance level of $a=0.05$, and were performed using R software, version 3.6 .0 (R Foundation for Statistical Computing).

Economic analyses were conduct with data from baseline and 36 month 24 -hour recall only. The cost-effectiveness ratios (CER) were calculated with the following equations:

Where $C E R^{Z}{ }_{i}=$ cost-effectiveness ratio of patient $i$ in group $Z ; C^{Z}{ }_{i}=$ direct cost of the patient's $i$ diet; $E^{Z}{ }_{i}=$ diet quality index of the patient's $i$ diet (BHEI-R). Direct cost was defined as:

Where $p_{i j}^{R}=$ means price of food item $j$ in region of residence $R$ of the patient $i ; q_{i j}^{R}=$ amount consumed of food item $j$ of the patient $i$ living in region $R$. Finally:

Where ICER = incremental cost-effectiveness ratio of group intervention in comparison to group control; $C_{m}^{\prime}=$ mean direct diet cost in intervention group; $C_{m}^{C}=$ mean direct diet cost in control group; $E_{m}^{\prime}=$ mean diet quality in intervention group; $E_{m}^{C}=$ mean diet quality in control group .

In addition, Monte Carlo simulations were performed to confirm trends in the comparison between control and intervention groups, resulting in 10,000 cases of patients in the intervention group and 10,000 cases of patients in the control group. Estimations on costs and effectiveness were based on mean and variance of direct costs and diet quality within each group using g distribution. The average of expected costs and effects were used to estimate different ICER for diverse scenarios, plotted onto incremental cost-effectiveness diagrams, with costs plotted in the horizontal axis and effects in the vertical axis.

\section{Results}

\section{Baseline characteristics}

Costs and BHEI-R were assessed for 1,161 participants (BALANCE Program $n=576$, Control $n=585$ ) of the study at baseline. Baseline characteristics were similar in both groups, as presented in Table 1. Overall, coronary artery disease was by far the most present cardiovascular disease. On average, participants were 63.5 years old and $59.1 \%$ were male. More than half of participants had less than 5 years of education and low household income (socio-economic classes between low and lower middle). 
Table 1

Baseline characteristics of individuals with cardiovascular disease from a subsample of BALANCE Program study.

\begin{tabular}{|c|c|c|c|c|c|c|c|}
\hline \multirow[t]{2}{*}{ Characteristics } & \multicolumn{3}{|c|}{ BALANCE Program $(n=576)$} & \multicolumn{3}{|c|}{ Control $(n=585)$} & \multirow[t]{2}{*}{$P$ value } \\
\hline & & $\%$ & $\mathbf{N}$ & & $\%$ & $\mathbf{N}$ & \\
\hline Age (mean, SD) & $63.0 / 7.93$ & & & $62.8 / 7.99$ & & & 0.565 \\
\hline Male & & 59.7 & 344 & & 59.3 & 347 & 0.935 \\
\hline Socioeconomic class ${ }^{a}$ & & & & & & & 0.106 \\
\hline High & & 3.53 & 20 & & 3.68 & 21 & \\
\hline Upper middle & & 27.16 & 154 & & 30.35 & 173 & \\
\hline Lower middle & & 56.97 & 323 & & 57.37 & 327 & \\
\hline Low & & 12.35 & 70 & & 8.6 & 49 & \\
\hline Years of study & & & & & & & 0.540 \\
\hline$\leq 4$ years & & 27.1 & 152 & & 24.0 & 138 & \\
\hline 5 years & & 32.1 & 180 & & 34.8 & 200 & \\
\hline 9 years & & 12.1 & 68 & & 13.7 & 79 & \\
\hline 12 years & & 20.0 & 112 & & 17.9 & 103 & \\
\hline$\geq 12$ years & & 8.73 & 49 & & 9.57 & 55 & \\
\hline Region of Brazil & & & & & & & 0.427 \\
\hline Northeast & & 23.8 & 137 & & 20.5 & 120 & \\
\hline North & & 6.08 & 35 & & 7.52 & 44 & \\
\hline Midwest & & 5.56 & 32 & & 5.98 & 35 & \\
\hline Southeast & & 35.8 & 206 & & 39.3 & 230 & \\
\hline South & & 28.8 & 166 & & 26.7 & 156 & \\
\hline \multicolumn{8}{|l|}{ Cardiovascular disease } \\
\hline Peripheral arterial disease & & 8.5 & 49 & & 9.7 & 57 & 0.529 \\
\hline Coronary artery disease & & 94.1 & 542 & & 93.5 & 547 & 0.766 \\
\hline Cerebrovascular disease & & 10.1 & 58 & & 8.9 & 52 & 0.557 \\
\hline Physical activity & & & & & & & 0.341 \\
\hline Sedentary & & 63 & 361 & & 59.79 & 348 & \\
\hline Active & & 37 & 212 & & 40.21 & 234 & \\
\hline Smoking & & & & & & & 0.764 \\
\hline Current & & 5.21 & 30 & & 6.15 & 36 & \\
\hline Former & & 56.6 & 326 & & 55.4 & 324 & \\
\hline Never & & 38.2 & 220 & & 38.5 & 225 & \\
\hline Weight (mean, SD) & $76.19 / 14.69$ & & & $76.15 / 14.91$ & & & 0.871 \\
\hline BMI (mean, SD) & $29.09 / 4.66$ & & & $28.98 / 4.86$ & & & 0.565 \\
\hline Waist circumference (mean, SD) & $99.81 / 11.94$ & & & $99.65 / 11.84$ & & & 0.788 \\
\hline
\end{tabular}

\section{Diet Quality And Costs}

At baseline, diet mean costs were identical (US\$3.7 per capital per day; $p$-value $=0.859)$ and BHEI-R were 53.5 and 51.8 points $(p=0.008)$ for intervention and control groups, respectively. After a three-year period, the intervention was associated with mean cost saving of US\$0.31 per capita per day ( $95 \% \mathrm{Cl}$ 
$-0.59 ;-0.04)$ and mean BHEI-R increase of 4.38 points $(95 \% \mathrm{Cl} 2.81 ; 5.95)($ Table 2$)$.

Table 2

Direct cost and Brazilian Healthy Eating Index Revised (BHEI-R) score per intervention period during follow-up of BALANCE Program study.

\begin{tabular}{|c|c|c|c|c|c|c|c|}
\hline \multirow[t]{3}{*}{ Cost (U\$) } & \multicolumn{2}{|c|}{ BALANCE Program } & \multicolumn{5}{|l|}{ Control } \\
\hline & \multirow[t]{2}{*}{ Mean } & \multirow[t]{2}{*}{$S D^{a}$} & \multirow[t]{2}{*}{ Mean } & \multirow[t]{2}{*}{ SD } & Difference & $95 \% \mathrm{Cl}^{\mathrm{b}}$ & $P$ value \\
\hline & & & & & \multicolumn{2}{|l|}{ BALANCE - Control } & \\
\hline Baseline & $3.7(n=575)$ & 1.7 & $3.7(n=585)$ & 2.0 & -0.02 & $-0.23 ; 0.19$ & 0.859 \\
\hline 12 months & $3.5(n=551)$ & 2.1 & $3.6(n=553)$ & 2.5 & -0.08 & $-0.35 ; 0.19$ & 0.547 \\
\hline 24 months & $3.4(n=498)$ & 1.9 & $3.6(n=510)$ & 2.1 & -0.26 & $-0.5 ;-0.01$ & 0.041 \\
\hline 36 months & $3.5(n=471)$ & 1.9 & $3.8(n=472)$ & 2.5 & -0.31 & $-0.59 ;-0.04$ & 0.027 \\
\hline \multicolumn{8}{|l|}{ BHEI-R } \\
\hline Baseline & $53.5(n=575)$ & 10.7 & $51.8(n=585)$ & 11.3 & 1.71 & $0.44 ; 2.9$ & 0.008 \\
\hline 12 months & $56.8(n=551)$ & 11.5 & $51.5(n=553)$ & 12.5 & 5.33 & $3.92 ; 6.74$ & $<0.001$ \\
\hline 24 months & $55.7(n=498)$ & 12.4 & $51.4(n=510)$ & 13 & 4.34 & $2.79 ; 5.89$ & $<0.001$ \\
\hline 36 months & $55.9(n=471)$ & 12.5 & $51.7(n=472)$ & 12.5 & 4.38 & $2.81 ; 5.95$ & $<0.001$ \\
\hline \multicolumn{8}{|c|}{ a Standard deviation. } \\
\hline b $95 \%$ cc & e interval. & & & & & & \\
\hline
\end{tabular}

Table 3 shows that there were increases in diet quality scores for the BALANCE group regarding total and whole fruits, total and dark green and orange vegetables; oil; saturated fat and solid fat; alcohol and added sugar (SoFAAS). Additionally, there were decreases in total energy, energy density and macronutrients, fat and carbohydrates without significant modifications in the amount consumed.

\section{Discussion}

This study presented a cost-effectiveness analysis of a randomised clinical trial, to assess the economic feasibility of nutritional intervention (BALANCE Program) designed for secondary prevention focusing on the adoption of regional foods in dietary prescription. After three years of follow-up participants from the intervention group of BALANCE program presented a significant improvement in diet quality (+ 4.38 points in BHEI-R) while saving U\$0.31 per day. Also, ICER probabilistic analyses showed that the BALANCE program was considered dominant in comparison to traditional counseling (-U\$0.08 per point in BHEI-R).

Dietary scores assess the adherence of individuals' or populations' intake to a certain nutritional recommendation. The index chosen in the study, the BHEI-R, comprises an adaptation of the Health Eating Index (HEI-2005) developed in the U.S. for the Brazilian population. Several studies assessed the association between $\mathrm{HEl}$ scores and diet costs. A recent meta-analysis found no significant differences in prices (\$1.61 international per capita per day, $95 \% \mathrm{Cl}-0.61 ; 3.84)$ between diets considered healthy and unhealthy according to the HEl-2005 score ${ }^{(15)}$. Our study found opposite results for the Brazilian diet quality indicator, showing that participants with higher BHEI-R score spent less money during the follow-up.

On the other hand, the same meta-analysis found that food intake according to Mediterranean dietary patterns became more expensive (\$1.18 international per capita per day, $95 \% \mathrm{Cl} 0.01 ; 2.36)^{(15)}$. A Spanish study found that a greater adherence to a Mediterranean dietary pattern was associated with higher spending on foods: daily cost was $+\$ 0.72$ euros for the highest diet scores ${ }^{(28)}$. Individuals who adhered to the Mediterranean diet spent more on fish, milk and dairy products, fruits and vegetables ${ }^{(28)}$. Monsivais et al. indicated that Americans with higher adherence to the Dietary Approaches to Stop Hypertension (DASH) pattern had an additional $20 \%$ in food spending ${ }^{(13)}$.

The BALANCE group showed better food quality after 36 months of follow-up mainly due to improvements in fruits, vegetables and fat components of BHEI-R score. Besides, was observed a reduction in calorie consumption without a decrease in the total amount of food consumed indicating less energy per gram (energy density). Following the nutritional recommendation of the BALANCE Program (21), which suggests increasing intake of green's group food (comprising of fruit, vegetables, legumes, skimmed milk, etc.) and to reduce intake of blue's food (comprising of meat, egg, cheese, etc.).

BHEI-R index does not permit a detailed analysis that shows which specific food or food group was able to contribute to the reduction in cost observed in BALANCE group. This is because scores of BHEI-R components are not directly correspondent to food amounts ${ }^{(27)}$. Thus, we conducted an exploratory analysis (supplementary table 4) to investigated which food group may have contributed to the reduction in food cost. There was a significant increase in intake (in grams) of milk, vegetables, fruits and a reduction in meat intake in the BALANCE group. However, the difference in cost was not significant in any cited groups. Highlighting, the average daily spending on meat (beef, pork, chicken and fish) and eggs that were lower -U\$ $0.15 ; 95 \% \mathrm{Cl}-0.33$ to 0.03 ) in the BALANCE group. Indeed, meat and fish are among the highest-priced foods in an analysis of the food purchase price in Brazil (29).

Page 6/11 
This randomized clinical trial is the first economic evaluation of longitudinal data considering food expenditures estimated from 24-hour recalls from participants with established cardiovascular disease in a developing country. There is a lack of economic assessment studies including intermediate clinical outcomes (e.g., diet quality, fruit and vegetable intake, weight or BMI) as parameters for effectiveness in cost-effectiveness analysis due to limitations regarding comparison with other interventions(30). However, the purpose of this study was to compare intervention and control groups to analyse if adherence to a cardioprotective diet in the Brazilian context can be affordable for low income patients.

The study has some limitations regarding data representativeness. The main limitation was the use of only one 24-hour food recall per period of followup, which may not reliably estimate the usual intake of a single individual. However, it is important to notice that it represents a valid estimate of the average dietary intake of a population ${ }^{(31)}$. A second limitation refers to the utilization of data from a subsample of the main research, comprising of a randomised controlled trial at the national level. In the present study, the objective was to analyze data from participants who engaged in at least $80 \%$ of the study protocol for both groups (intervention and control).

The guidelines for economic evaluations of clinical trials recommend considering intention-to-treat analyses (datasets from the original trial) to perform cost-effectiveness analysis(32). However, considering real world scenarios, neither costs nor benefits are incurred by non-participants ${ }^{(24)}$. In addition, the perspective adopted in the current study aimed to provide economic information useful at the patient level, considering that patients decide whether to adopt dietary prescription or not. Furthermore, the selection of a subsample including only individuals with higher adherence for the economic analysis was performed to allow the comparison of information throughout the period of follow-up, in order to exclude individuals with incomplete data. The descriptive analyses showed that the random effect was maintained in the subsample because the characteristics of individuals regarding the eligibility criteria remained the same between groups.

\section{Conclusion}

The BALANCE program intervention group showed statistically significant improvements in diet quality with lower costs following three-year dietary guidance, indicating that the intervention is dominant in terms of cost-effectiveness in comparison to the control group (traditional counseling). The findings may be used by health professionals to encourage the adherence and recommendations of BALANCE nutritional education strategy, which showed higher diet quality with lower costs in comparison to the typical Brazilian diets for low-income individuals in secondary prevention programs at the primary health care level.

\section{List Of Abbreviations}

CVD -Cardiovascular disease

BALANCE Program -Brazilian Cardioprotective Nutritional Program Trial

CER- cost-effectiveness ratios

ICER- incremental cost-effectiveness ratios

BHEI-R-Brazilian Health Eating Index Revised

IBGE- Brazilian Institute for Geography and Statistics

SoFAAS- solid fat, alcohol, and added sugar

GEE -generalized estimating equation

HEl-2005 -Health Eating Index

DASH- Dietary Approaches to Stop Hypertension

\section{Declarations}

This manuscipt adheres to CHEERS guidelines for economic evaluations.

\section{Ethics approval and consent to participate}

The study was approved by the Research Ethics Committee of the Hospital do Coração de São Paulo and all participants signed an informed consent form.

\section{Consent for publication}

Not applicable 


\section{Availability of data and materials}

The datasets used and/or analysed during the current study are available from the corresponding author on reasonable request.

\section{Competing interests}

The authors declare that they have no competing interests.

\section{Funding}

The BALANCE trial and this sub analyses were supported by the Program to Support Institutional Development of the Brazilian Healthcare System from the Brazilian Ministry of Health.It financed patients' transport and blood tests, educational materials and researchers responsible for conducting the study and analyzing data.

\section{Authors' contributions}

All authors have read and approved the manuscript.

CRT

Made substantial contributions to the conception, analyses and interpretation of data for the work. Wrote and reviewed the paper.

FMS

Made substantial contributions to the conception. Reviewed the paper

ÂCBF

Made substantial contributions to the analyses and interpretation of data for the work. Reviewed the paper.

BW

Made substantial contributions to the conception and reviewed the paper.

RHNS

Made substantial contributions to the analyses and interpretation of data for the work.

MMS

Made substantial contributions to the analyses and interpretation of data for the work.

ADPCF

Made substantial contributions to the conception. Reviewed the paper

\section{Acknowledgements}

We thank Messilyana de Oliveira Mesquita, Olivia Farias dos Santos, Amanda de Oliveira Guimarães who work in data price collect.

The author declares that permissions for acknowledgements from Messilyana de Oliveira Mesquita, Olivia Farias dos Santos and Amanda de Oliveira Guimarães were obtained.

\section{References}

1. Joseph P, Leong D, McKee M, Anand SS, Schwalm J-D, Teo K, et al. Reducing the Global Burden of Cardiovascular Disease, Part 1. Circ Res [Internet]. 2017 Sep 1 [cited 2019 Jul 6];121(6):677-94. Available from: http://www.ncbi.nlm.nih.gov/pubmed/28860318.

2. 10.1161/CIRCULATIONAHA.114.008727 Ribeiro ALP, Duncan BB, Brant LCC, Lotufo PA, Mill JG, Barreto SM. Cardiovascular Health in Brazil. Circulation [Internet]. 2016;133(4):422-33. Available from: http://circ.ahajournals.org/lookup/doi/10.1161/CIRCULATIONAHA.114.008727.

3. Clark AM, Hartling L, Vandermeer B, McAlister FA. Meta-Analysis: Secondary Prevention Programs for Patients with Coronary Artery Disease. Ann Intern Med [Internet]. 2005 Nov 1 [cited 2019 May 5];143(9):659. Available from: http://annals.org/article.aspx?doi=10.7326/0003-4819-143-9- 
200511010-00010.

4. Clara K Epidemiology and Prevention Association of Diet, Exercise, and Smoking Modification With Risk of Early Cardiovascular Events After Acute Coronary Syndromes. 2010 [cited 2019 Feb 9]; Available from: http://circ.ahajournals.org.

5. the Preventive Cardiovascular Nurses Association

Smith SC, Benjamin EJ, Bonow RO, Braun LT, Creager MA, Franklin BA, et al. AHA/ACCF Guideline AHA/ACCF Secondary Prevention and Risk Reduction Therapy for Patients With Coronary and Other Endorsed by the World Heart Federation and the Preventive Cardiovascular Nurses Association. 2011 [cited 2019 Feb 9]; Available from: http://circ.ahajournals.org.

6. Piepoli MF, Hoes AW, Agewall S, Albus C, Brotons C, Catapano AL, et al. 2016 European Guidelines on cardiovascular disease prevention in clinical practice. Eur Heart J. 2016;37(29):2315-81.

7. Cole JA, Smith SM, Hart N, Cupples ME. Systematic review of the effect of diet and exercise lifestyle interventions in the secondary prevention of coronary heart disease. Cardiol Res Pract [Internet]. 2010 Dec 19 [cited 2019 May 6];2011:232351. Available from:

http://www.ncbi.nlm.nih.gov/pubmed/21197445.

8. Mozaffarian D. Dietary and Policy Priorities for Cardiovascular Disease, Diabetes, and Obesity - A Comprehensive Review. Circulation [Internet]. 2016 Jan 12 [cited 2019 Jul 6];133(2):187. Available from: http://www.ncbi.nlm.nih.gov/pubmed/26746178.

9. Schwingshackl L, Schwedhelm C, Hoffmann G, Lampousi A-M, Knüppel S, lqbal K, et al. Food groups and risk of all-cause mortality: a systematic review and meta-analysis of prospective studies. Am J Clin Nutr [Internet]. 2017 Apr 26 [cited 2019 Jul 6];105(6):ajcn153148. Available from: https://academic.oup.com/ajcn/article/105/6/1462-1473/4569801.

10. Dehghan M, Mente A, Zhang X, Swaminathan S, Li W, Mohan V, et al. Associations of fats and carbohydrate intake with cardiovascular disease and mortality in 18 countries from five continents (PURE): a prospective cohort study. Lancet (London, England) [Internet]. 2017 Nov 4 [cited 2019 Jul 6];390(10107):2050-62. Available from: http://www.ncbi.nlm.nih.gov/pubmed/28864332.

11. $10.1111 /$ obr.12078

Lee A, Mhurchu CN, Sacks G, Swinburn B, Snowdon W, Vandevijvere S, et al. Monitoring the price and affordability of foods and diets globally. 2013 [cited 2018 Oct 12]; Available from: https://onlinelibrary.wiley.com/doi/pdf/10.1111/obr.12078.

12. Mozaffarian D, Angell SY, Lang T, Rivera JA. Role of government policy in nutrition-barriers to and opportunities for healthier eating. BMJ [Internet]. 2018 Jun 13 [cited 2019 Jul 6];361:k2426. Available from: http://www.ncbi.nlm.nih.gov/pubmed/29898890.

13. Monsivais P, Rehm CD, Drewnowski A. The DASH diet and diet costs among ethnic and racial groups in the United States. JAMA Intern Med [Internet]. 2013 Nov 11 [cited 2019 Jul 6];173(20):1922-4. Available from: http://www.ncbi.nlm.nih.gov/pubmed/23999924.

14. Tong TYN, Imamura F, Monsivais P, Brage S, Griffin SJ, Wareham NJ, et al. Dietary cost associated with adherence to the Mediterranean diet, and its variation by socio-economic factors in the UK Fenland study Europe PMC Funders Group. Br J Nutr [Internet]. 2018 [cited 2018 Oct 12];119(6):685-94. Available from: https://www.ncbi.nlm.nih.gov/pmc/articles/PMC5999016/pdf/emss-77689.pdf.

15. 10.1136/

Rao M, Afshin A, Singh G, Mozaffarian D. Do healthier foods and diet patterns cost more than less healthy options? A systematic review and metaanalysis [Internet]. BMJ Open 2013. Available from: http://dx.doi.org/10.1136/.

16. Reidlinger DP, Sanders TA, Goff LM. How expensive is a cardioprotective diet? Analysis from the CRESSIDA study. Public Health Nutr [Internet]. 2017;20(08):1423-30. Available from:

http://www.ncbi.nlm.nih.gov/pubmed/28095936\%5Cnhttps://www.cambridge.org/core/product/identifier/S1368980016003529/type/journal_article.

17. Jensen JD, Poulsen SK. The new nordic diet - Consumer expenditures and economic incentives estimated from a controlled intervention. BMC Public Health. 2013.

18. Thomazella MCD, Goes MFS, Andrade CRCR, Debbas V, Barbeiro DF, Correia RL, et al. Effects of high adherence to mediterranean or low-fat diets in medicated secondary prevention patients. Am J Cardiol [Internet]. 2011;108(11):1523-9. Available from: http://www.scopus.com/inward/record.url? eid=2-s2.0-80955163668\&partnerlD=tZOtx3y1.

19. Weber B, Galante AP, Bersch-Ferreira AC, Torreglosa CR, Carvalho VO, Victor E da. S, et al. Effects of Brazilian Cardioprotective Diet Program on risk factors in patients with coronary heart disease: A Brazilian Cardioprotective Diet randomized pilot trial. Clinics. 2012;67(12):1407-14.

20. 10.1016/j.ahj.2015.08.010

Weber B, Bersch-Ferreira ÂC, Torreglosa CR, Ross-Fernandes MB, Da Silva JT, Galante AP, et al. The Brazilian Cardioprotective Nutritional Program to reduce events and risk factors in secondary prevention for cardiovascular disease: study protocol (The BALANCE Program Trial) Trial Designs. Am Heart J [Internet]. 2016 [cited 2018 Oct 9];171:73-81.e2. Available from: http://dx.doi.org/10.1016/j.ahj.2015.08.010.

21. Weber B, Bersch-Ferreira ÂC, Torreglosa CR, Marcadenti A, Lara ES, da Silva JT, et al. Implementation of a Brazilian Cardioprotective nutritional (BALANCE) program for improvement on quality of diet and secondary prevention of cardiovascular events: A randomized, multicentre trial. Am Heart J [Internet]. 2019 Jun 21 [cited 2019 Jul 2]; Available from: https://www.sciencedirect.com/science/article/pii/S0002870319301553.

22. ON CARDIOVASCULAR PREVENTION [Internet] Brasileira De Cardiologia S. I BRAZILIAN GUIDELINES. ON CARDIOVASCULAR PREVENTION [Internet]. Vol. 101. 2013 [cited 2019 Jul 6]. Available from: .

23. Bouchard DR, Baillargeon JP, Gagnon C, Brown C, Langlois MF. Impact of health professionals' contact frequency on response to a lifestyle intervention with individuals at high risk for diabetes. Diabetes Res Clin Pract [Internet]. 2012 May [cited 2020 Mar 29];96(2):129-34. Available from:

Page $9 / 11$ 
http://www.ncbi.nlm.nih.gov/pubmed/22245692.

24. Herman WH, Edelstein SL, Ratner RE, Montez MG, Ackermann RT, Orchard TJ, et al. Effectiveness and cost-effectiveness of diabetes prevention among adherent participants. Am J Manag Care. 2013 Mar;19(3):194-202.

25. Previdelli ÀN, Andrade SC, De, Pires MM, Fereira SRG, Fisberg RM, Marchioni DM. Índice de Qualidade da Dieta Revisado para população brasileira A revised version of the Healthy Eating Index for the Brazilian. Rev Saúde Pública. 2011;45(4):794-8.

26. E IB de G e E. Índice Nacional de Preços ao Consumidor Amplo - IPCA e Índice Nacional de Preços ao Consumidor - INPC [Internet]. Índice de Preços ao Consumidor Amplo. Available from: https://www.ibge.gov.br/estatisticas/economicas/precos-e-custos/9256-indice-nacional-de-precos-aoconsumidor-amplo.html?=\&t=o-que-e.

27. Guenther PM, Reedy J, Krebs-Smith SM. Development of the Healthy Eating Index-2005. J Am Diet Assoc [Internet]. 2008 Nov [cited 2019 Jul 6];108(11):1896-901. Available from: http://www.ncbi.nlm.nih.gov/pubmed/18954580.

28. Schröder H, Gomez SF, Ribas-Barba L, Pérez-Rodrigo C, Bawaked RA, Fíto M, et al. Monetary Diet Cost, Diet Quality, and Parental Socioeconomic Status in Spanish Youth. PLoS One [Internet]. 2016 [cited 2019 Aug 11];11(9):e0161422. Available from: http://www.ncbi.nlm.nih.gov/pubmed/27622518.

29. Claro RM, Maia EG, Costa BV, de L, Diniz. DP. Food prices in Brazil: prefer cooking to ultra-processed foods. Cad Saude Publica. 2016 Aug 29;32(8):e00104715.

30. Cost-Effectiveness in. Health and Medicine. Cost-Effectiveness in Health and Medicine. Oxford University Press; 2016.

31. Baranowski T. 24-Hour Recall and Diet Record Methods. In: Nutritional Epidemiology. Oxford University Press; 2013.

32. Ramsey SD, Willke RJ, Glick H, Reed SD, Augustovski F, Jonsson B, et al. Cost-effectiveness analysis alongside clinical trials II - An ISPOR good research practices task force report. Value Heal. 2015 Mar 1;18(2):161-72.

\section{Figures}

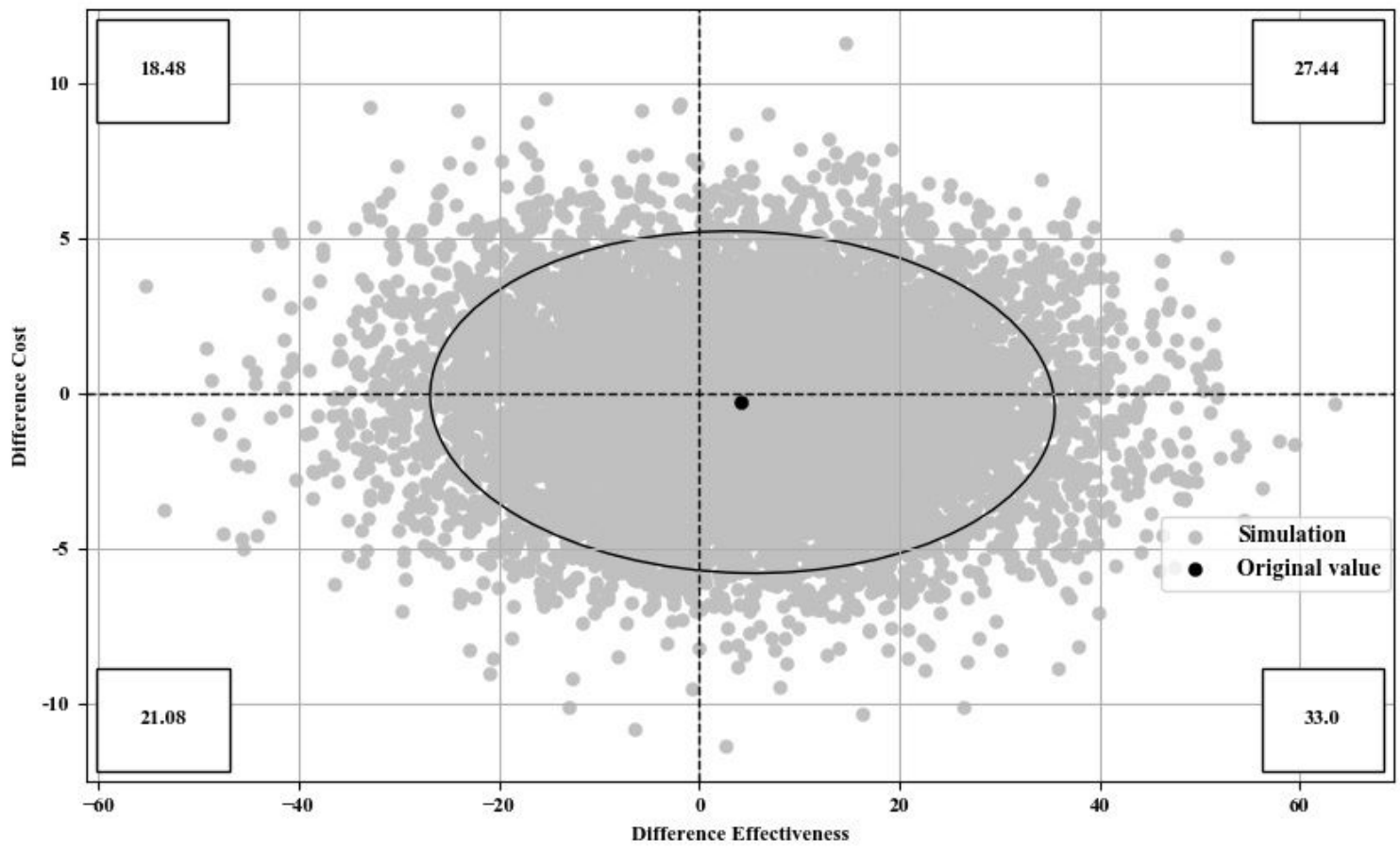

Figure 1

Incremental cost-effectiveness diagram at baseline. Brazil, 2013-2015. Simulation: ICER = incremental cost-effectiveness of intervention group versus control group (10,000 cases for each group); Original value: ICER = incremental cost-effectiveness ratio (-U\$0.01 per BHEI-R point). 


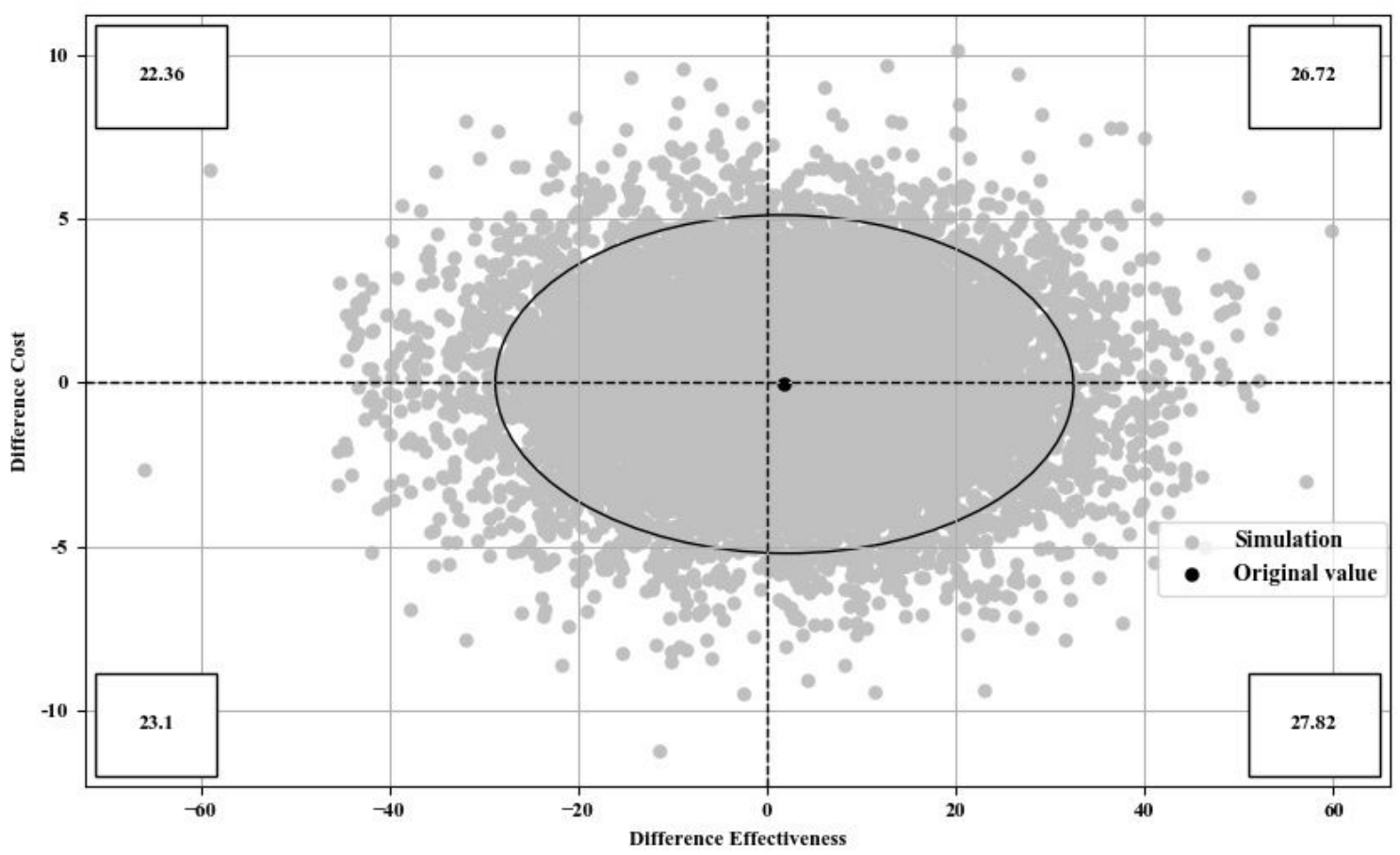

\section{Figure 2}

Incremental cost-effectiveness diagram at 36 months of follow up. Brazil, 2013-2017. Simulation: ICER = incremental cost-effectiveness of intervention group versus control group (10,000 cases for each group); Original value: ICER = incremental cost-effectiveness ratio (-U\$ 0.08 per BHEI-R point). 